\title{
Attenuation of Sinapic Acid and Sinapine-Derived Flavor-Active Compounds Using A Factorial-Based Pressurized High-Temperature Processing
}

\author{
Ruchira Nandasiri ${ }^{1}$, Erika Zago ${ }^{1}$, Usha Thiyam-Holländer ${ }^{1}$, and Michael Eskin ${ }^{1}$ \\ ${ }^{1}$ University of Manitoba Faculty of Agricultural and Food Sciences
}

March 28, 2021

\begin{abstract}
De-oiled canola meals are sources of protein-containing flavor-active phenolic compounds. Conventional canola oil processing utilizes an excess amount of solvents and is associated with the release of high-intensity bitter flavor-active phenolic compounds, limiting the use of the canola meal. Recent advances in the extraction and isolation of the bitter favor-active phenolic compounds from canola by-products produce protein isolates, however, would benefit the industry by producing a side-stream ingredient rich in phenolics. High temperature and pressure-aided processing, namely the accelerated solvent extraction (ASE) was investigated to extract the flavor-active bitter molecules from the canola meal. The extractability of flavor-active phenolic compounds including the major sinapates, kaempferol derivatives, and other thermo-generative compounds including thomasidioc acid (TA) was evaluated. The effects of temperature, solvent extractant and concentration, and the particle size of the meal, were examined on the extraction efficiency of these phenolic compounds. Extraction temperature (180oC) was the primary determinant $(\mathrm{p}<0.05)$ for the attenuation of major sinapates including sinapine and sinapic acid. Both ethanol and methanol extractants at a concentration of $70 \%(\mathrm{v} / \mathrm{v})$ significantly $(\mathrm{p}<0.05)$ extracted the flavor-active phenolic compounds. The pressurized high temperature through optimized ASE conditions attenuated the bitter undesirable flavor-active phenolic molecules from canola meal thereby facilitating a potential value-added phenolic-rich by-product.
\end{abstract}

\section{Hosted file}

Cover letter_JAOCS_25032021.pdf available at https://authorea.com/users/404523/articles/ 515688-attenuation-of-sinapic-acid-and-sinapine-derived-flavor-active-compounds-using-afactorial-based-pressurized-high-temperature-processing

\section{Hosted file}

Reviewers comments_09032021.pdf available at https://authorea.com/users/404523/articles/ 515688-attenuation-of-sinapic-acid-and-sinapine-derived-flavor-active-compounds-using-afactorial-based-pressurized-high-temperature-processing

\section{Hosted file}

JAOCS_Manuscript_Modified_08032021.pdf available at https://authorea.com/users/404523/ articles/515688-attenuation-of-sinapic-acid-and-sinapine-derived-flavor-activecompounds-using-a-factorial-based-pressurized-high-temperature-processing

\section{Hosted file}

Figure 1.pdf available at https://authorea.com/users/404523/articles/515688-attenuationof-sinapic-acid-and-sinapine-derived-flavor-active-compounds-using-a-factorial-basedpressurized-high-temperature-processing 


\section{Hosted file}

Table 1.pdf available at https://authorea.com/users/404523/articles/515688-attenuationof-sinapic-acid-and-sinapine-derived-flavor-active-compounds-using-a-factorial-basedpressurized-high-temperature-processing

\section{Hosted file}

Table 2.pdf available at https://authorea.com/users/404523/articles/515688-attenuationof-sinapic-acid-and-sinapine-derived-flavor-active-compounds-using-a-factorial-basedpressurized-high-temperature-processing

\section{Hosted file}

Table 3.pdf available at https://authorea.com/users/404523/articles/515688-attenuationof-sinapic-acid-and-sinapine-derived-flavor-active-compounds-using-a-factorial-basedpressurized-high-temperature-processing

\section{Hosted file}

Table 4.pdf available at https://authorea.com/users/404523/articles/515688-attenuationof-sinapic-acid-and-sinapine-derived-flavor-active-compounds-using-a-factorial-basedpressurized-high-temperature-processing

\section{Hosted file}

Table 5.pdf available at https://authorea.com/users/404523/articles/515688-attenuationof-sinapic-acid-and-sinapine-derived-flavor-active-compounds-using-a-factorial-basedpressurized-high-temperature-processing 